\title{
Compressible Gas-Solid Mixture Conservation Laws Simulations
}

\author{
D. Zeidan* ${ }^{*}$ A. Slaouti ${ }^{\dagger}$ and R. Touma** \\ *Department of Fundamental \& Applied Sciences, Universiti Teknologi PETRONAS, Perak, Malaysia \\ ${ }^{\dagger}$ School of Engineering, University of Newcastle Australia, Singapore \\ ** Department of Computer Science \& Mathematics, Lebanese American University, Beirut, Lebanon
}

\begin{abstract}
Computations of gas-solid two-phase flow are carried out within the framework of mixture formulations. These computations are based on the Riemann problem for the resulting theoretical equations. The Riemann problem for the model equations is solved numerically using Godunov methods of centred-type. Numerical results are shown for carefully chosen test problems. The results show that both the model and the numerical methods provide very satisfactory solutions.
\end{abstract}

Keywords: gas-solid, compressible mixture, hyperbolic conservative equations, Riemann problem

PACS: $47.40 . \mathrm{Nm}, 47.11 .+\mathrm{j}, 47.55 .-\mathrm{t}$

\section{INTRODUCTION}

Together with numerical analysis, two-phase fluid flow problems remain one of the most challenging fields of applied mathematics. Two-phase flows have application in many areas of science, engineering and technology, and the equations that describe them pose theoretical and computational challenges. Such challenges are related to the hyperbolicity and conservativity character of the governing equations. Traditionally, formulations of two-phase fluid flow problems have been investigated by either the two-fluid models or the mixture models [1] which provided simplified models. Compressible gas-solid mixture problems are encountered in a wide range of areas: volcanic eruptions, explosions in grain storage and transportation, combustion in porous media etc. Most current gas-solid models are used with a certain degree of success but are inherently formulated in a non-conservative form. Amongst formulations and computations for simulating two-phase flows is the mixture formulation with velocity non-equilibrium between phases [2]. The resulting system of models using such an approach is fully hyperbolic and fully conservative. This paper describes one of these theoretical models. The model considered in the current paper describes a one-dimensional flow of gas and solid mixture completed by additional closure governing equations. The mixture formulation of gas-solid mixture equations, where the velocity and pressure are in non-equilibrium, is considered as follows:

$$
\frac{\partial}{\partial t}\left(\begin{array}{c}
\rho \\
\rho u \\
\rho E \\
\rho \alpha \\
\rho c \\
u_{r}
\end{array}\right)+\frac{\partial}{\partial x}\left(\begin{array}{c}
\rho u \\
\rho u^{2}+P+\rho c(1-c) u_{r}^{2} \\
\rho u E+P u+\rho c(1-c) u_{r}\left(u u_{r}+(1-2 c) \frac{u_{r}^{2}}{2}+\frac{\partial e}{\partial c}\right) \\
\rho u \alpha \\
\rho u c+\rho c(1-c) u_{r} \\
u u_{r}+(1-2 c) \frac{u_{r}^{2}}{2}+\frac{\partial e}{\partial c}
\end{array}\right)=\left(\begin{array}{l}
0 \\
0 \\
0 \\
\phi \\
\mathcal{C} \\
\pi
\end{array}\right) .
$$

The first three equations represent the mixture conservation equations for mass, momentum and energy. The fourth and the fifth conservation equations refer to the volume fraction and mass fraction for the gas phase and the last equation represent the relative velocity between the gas and solid phases. In addition to that, the source terms on the right hand side represent the interaction between the gas and solid phases. While not shown here, the first and second source terms are similar to those used within the detonation community and the third source term is similar to the interphase drag. Finally, system (1) is fully conservative and fully hyperbolic with velocity non-equilibrium between the gas phase and solid phase.

Numerical Analysis and Applied Mathematics ICNAAM 2012

AIP Conf. Proc. 1479, 165-168 (2012); doi: 10.1063/1.4756088

(C) 2012 American Institute of Physics 978-0-7354-1091-6/\$30.00 


\section{COMPUTATIONAL PROCEDURE}

The system, (1), is solved numerically using a finite volume discretization at cell interface

$$
\mathbb{U}_{i}^{n+1}=\mathbb{U}_{i}^{n}-\frac{\Delta t}{\Delta x}\left[\mathbb{F}_{i+\frac{1}{2}}-\mathbb{F}_{i-\frac{1}{2}}\right]+\Delta t \mathbb{S}_{i}
$$

The numerical flux functions $\mathbb{F}_{i \pm \frac{1}{2}}$ follow from the solution of the Riemann problems, system (1), subject to the initial conditions: $\mathbb{U}(x, 0)=\mathbb{U}_{L, i+\frac{1}{2}}$ if $x<x_{i+\frac{1}{2}}$, and $\mathbb{U}(x, 0)=\mathbb{U}_{R, i+\frac{1}{2}}$ if $x>x_{i+\frac{1}{2}}$, where $\mathbb{U}_{L, i+\frac{1}{2}}$ and $\mathbb{U}_{R, i+\frac{1}{2}}$ are discontinuous left and right states on either side of the interface. The solution of the Riemann problem for the model consists of six wave patterns produced by the decomposition of the initial discontinuity. The two left and two right waves are shocks or rarefactions separated by multiple contact discontinuities moving at the mixture velocity. In general, the Riemann problem requires the solution of a non-linear algebraic system of equations written as a function of a single unknown quantity, which cannot be obtained analytically. This prevent us from using Godunov methods of upwind-type based on an algebraically given Riemann solver. Here a Godunov centred-type method, namely, the slope limiter centred (SLIC) scheme is used, where the solution of the Riemann problem is fully numerical. Such a method is overall explicit and its stability is controlled by the CFL criterion [2]. To demonstrate the validity and the accuracy of the model and the method, numerical results for shock-tube problems are presented. The first test problem, Test 1 , consists of four shock waves and multiple contact discontinuities, typical of a collision problem. The second test problem, Test 2 which is selected from [4], deals with two left rarefaction waves and two right shock waves for the mixture separated by multiple contact discontinuities. For all computations presented in this paper, the computational domain is chosen to be in the interval $[-10,10]$ and the discontinuity is located at $x_{0}=0$. The CFL number is set to 0.9 with transmissive boundary conditions together with the compressive SUPERBEE limiter. The reference solution in each plot is a high-resolution numerical solution on 3000 mesh cells by the TVD SLIC scheme. The numerical solutions are computed with 100 mesh cells using the TVD SLIC scheme. All the results are compared with an alternative numerical method such as the unstaggered central scheme (UCS) which is regarded as a Riemann-free solver [3]. Figures 1 and 3 display the solutions to the model equations without the source terms effect. In figures 2 and 4, the interphase drag in the relative velocity equations is taken into account to solve the full model equations. The drag force computation was taken from [4]. The TVD SLIC scheme obviously provides superior results in all of the two cases. Further, the main flow structure - rarefactions, shocks and contact discontinuities - are all evidently resolved with the TVD SLIC scheme. Good agreement is clearly shown with the reference solutions.
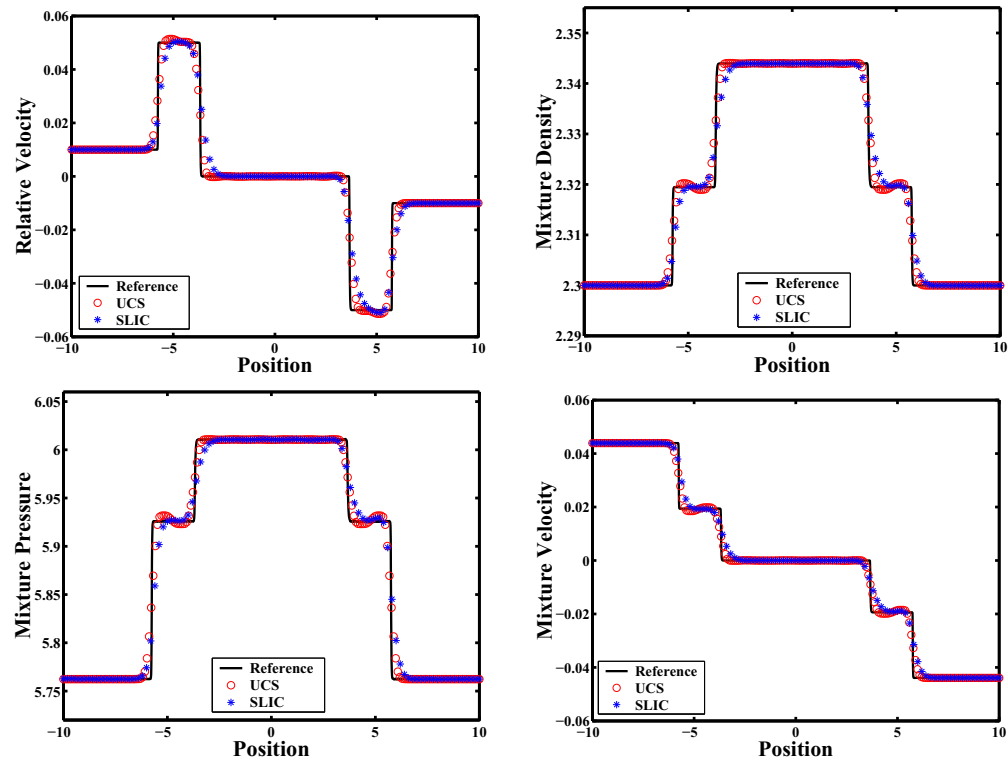

FIGURE 1. Results for Test 1 . The two different methods (TVD SLIC with CFL $=0.9$ and UCS with CFL $=0.485$ ) are compared with the reference solution (solid line) at time $t=2$. 

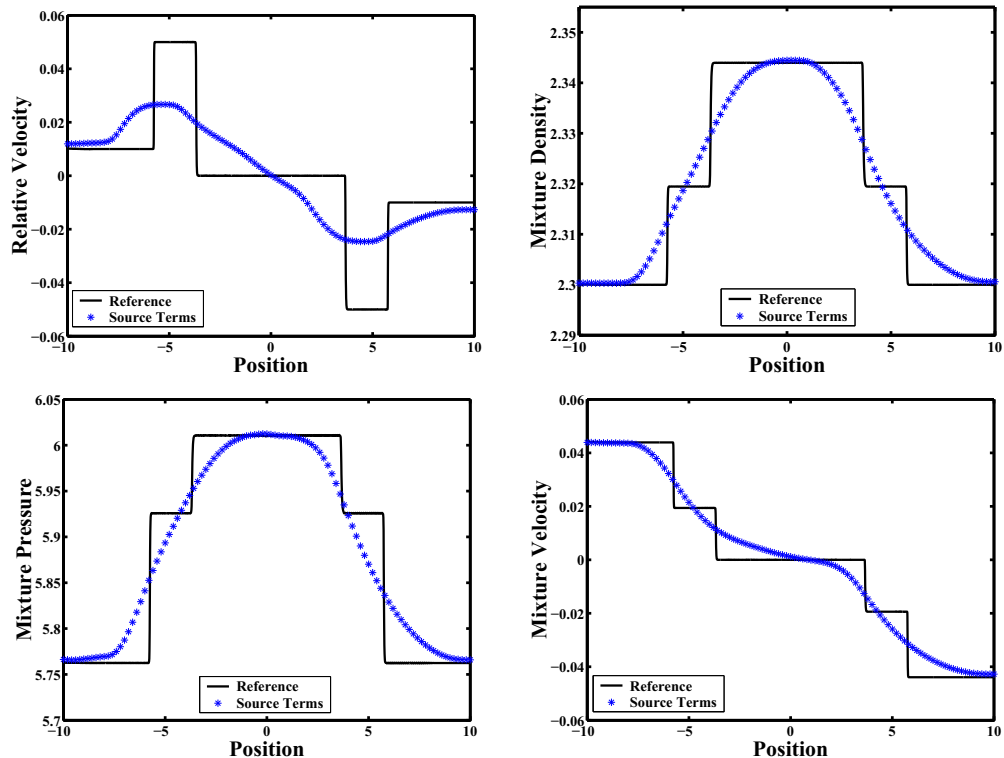

FIGURE 2. Results for Test 1 . Solutions of the full gas-solid mixture equations using the splitting approach along with the TVD SLIC scheme at $t=2$. The interphase drag force of [4] is considered with $C_{D}=0.44$.
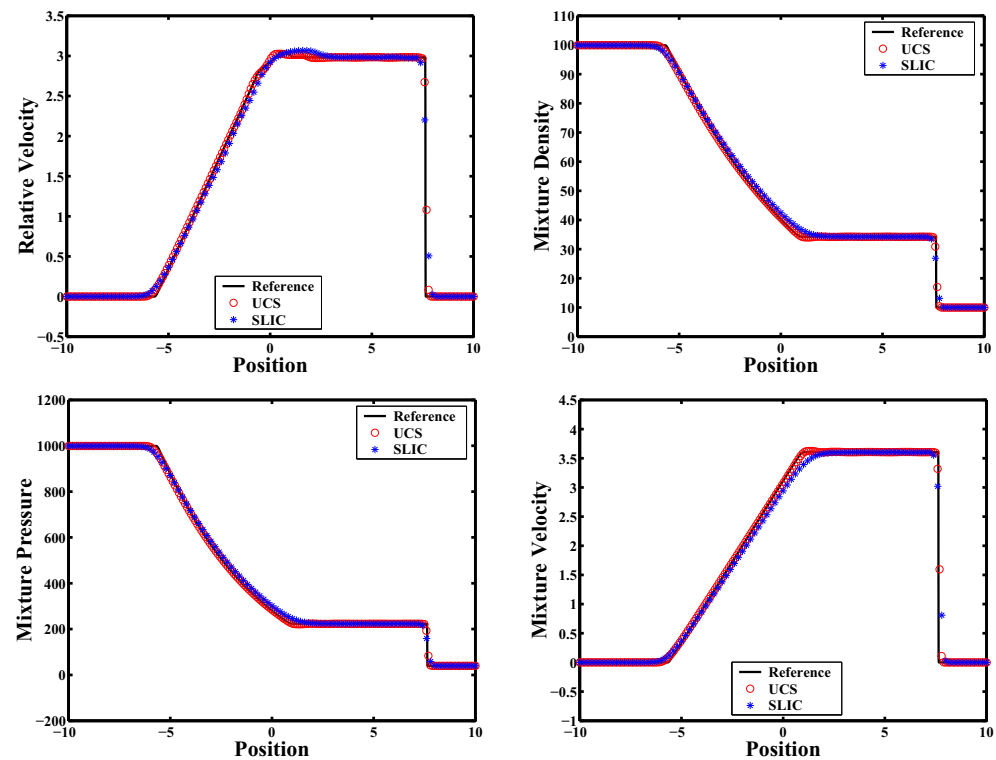

FIGURE 3. Results for Test 2. The TVD SLIC with CFL $=0.9$ and UCS with CFL $=0.485$ are compared with the reference solution (solid line) at time $t=1.5$. Depending on the initial left and right conditions of the Riemann problem, the solutions to the model equations develop left rarefaction waves and right shock waves separated by a contact discontinuity. 

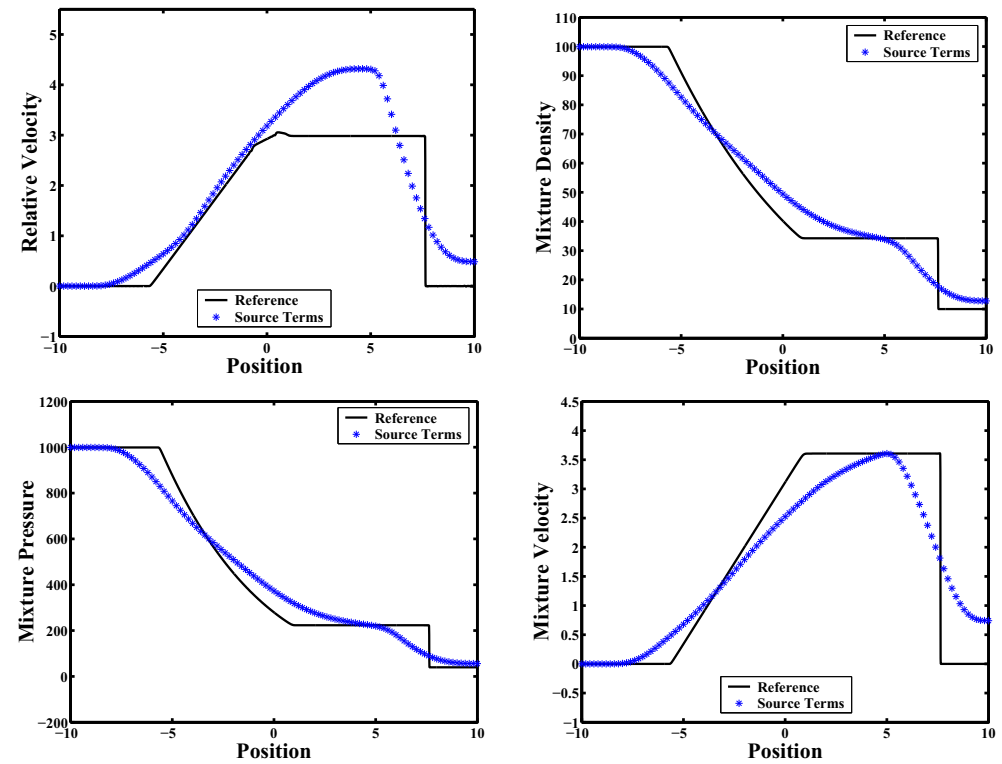

FIGURE 4. Results for Test 2. Effect of source terms of [4] on wave propagations within compressible gas-solid mixture at $t=1.5$.

\section{CONCLUDING REMARKS}

Accurate prediction of the solution of the Riemann problem for compressible gas-liquid mixture flow is presented. The obtained results suggest that a well-defined mathematical model based on mixture formulations leads to consistent and accurate simulation results for wave propagation. Further work calls into investigating the source terms behavior within non-equilibrium gas-solid mixtures in one and multiple dimensions.

\section{ACKNOWLEDGMENTS}

The authors gratefully acknowledge the financial support provided by the Universiti Teknologi PETRONAS.

\section{REFERENCES}

1. M. Ishii and T. Hibiki, Thermo-Fluid Dynamics of Two-Phase Flow. Springer, 2006

2. D. Zeidan, A. Slaouti, E. Romenski and E.F. Toro, Numerical Solution for Hyperbolic Conservative Two-Phase Flow Equations, International Journal of Computational Methods 4, 299-333 (2007).

3. R. Touma, Central Unstaggered Finite Volume Schemes for Hyperbolic Hystems: Applications to Unsteady Shallow Water Equations, Applied Mathematics and Computation, 213, 47-59 (2009).

4. T. Saito, M. Marumoto and K. Takayama, Numerical Investigations of Shock Waves in Gas-Particle Mixtures, Shock Waves 13, 299-322 (2003). 
Copyright of AIP Conference Proceedings is the property of American Institute of Physics and its content may not be copied or emailed to multiple sites or posted to a listserv without the copyright holder's express written permission. However, users may print, download, or email articles for individual use. 